\title{
A Centralized Feedback Control Model for Resource Management in Wireless Networks
}

\author{
Yimeng Yang, Boudewijn R. Haverkort, Geert J. Heijenk \\ Design and Analysis of Communication Systems, University of Twente \\ 7500 AE, Enschede, The Netherlands \\ \{y.yang, b.r.h.m.haverkort, geert.heijenk\}@utwente.nl
}

\begin{abstract}
In a wireless environment, guaranteeing QoS is challenging because applications at multiple devices share the same limited radio bandwidth. In this paper we introduce and study a resource management model for centralized wireless networks, using feedback control theory. Before applying in practice, the proposed model is evaluated using the well-known 20-sim dynamic system simulator. The experimental results show that flexible and efficient resource allocation can be achieved for a variety of system parameters and WLAN scenarios; however, care should be taken in setting the control parameters and coefficients.
\end{abstract}

\section{INTRODUCTION}

In recent years, a lot of research has been done in the area of wireless networks, and multimedia services such as data (non-real-time services), video, and voice (real-time services) have to be supported by future wireless systems [1]. Obviously, different services have different quality requirements (QoS), and in order to satisfy these, guaranteeing delay and bandwidth capacity is one of the key issues [2]. Therefore, searching for an efficient way to provide adaptable channel resource management is very important.

Control engineering is based on the foundations of feedback theory and linear system analysis, which has been widely applied in various areas for hundreds of years [3]. Recently, control theory has also been used in the context of wireless and wired communication networks, addressing issues like transmission power control and congestion control [4]. Besides these, channel resource management can also be defined as a control problem, thus control theoretic approaches would be applicable here as well.

In this paper, we will present a centralized feedback control model, in order to provide channel resource management in an efficient and flexible way, according to the current network scenario and different QoS requirements of different services. The paper is organized as follows. Section II introduces related work and analyzes the corresponding applicability. Section III presents a new feedback control model based on the centralized medium access method. Section IV presents first experimental results, and in Section V the paper is concluded.

\section{RELATED WORK AND APPLICABILITY ANALYSIS}

The basic IEEE 802.11 Medium Access Control (MAC) defines two forms of medium access, Distributed Coordination Function (DCF), which is mandatory and based on Carrier Sense Multiple Access with Collision Avoidance (CSMA/CA) protocol, and Point Coordination Function (PCF) providing the centrally controlled contention-free services. Compared to the 802.11 standard, the IEEE 802.11e enhances the DCF and the PCF, and provides QoS support for multimedia flows, which is critical for delay-sensitive applications. A dynamic bandwidth allocation scheme for IEEE 802.11e WLANs with the centralized medium access method HCCA (Hybrid-coordination function Controlled Channel Access) has been proposed in [5]. The IEEE 802.11e WLAN system is composed of an Access Point (AP) which can be seen as the central coordinator, and a set of QoS enabled mobile stations (QSTAs). Every QSTA has at most 4 queues, one for every Access Category (AC), acting as a Virtual Station (VS) with its own QoS parameters. The AP implements a medium access control under a superframe-based timing scheme. Within each superframe, the HCCA periods are organized periodically, and $T_{C A}$ is defined as the time interval between two successive HCCAs, which is assumed to be constant [5]. In this paper, we also address the control algorithm for centralized resource allocation; therefore, we only consider the HCCA process and a simplified timing scheme is given in Fig. 1.

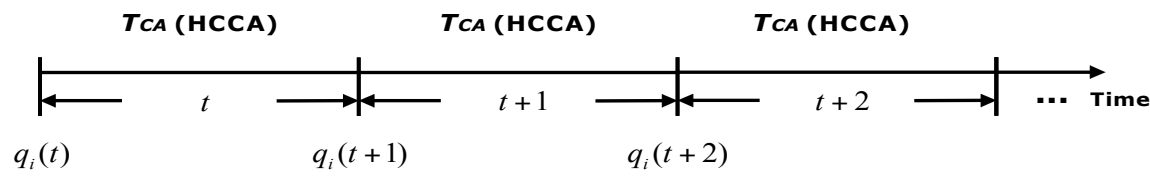

Fig. 1. A simplified expression of the HCCA timing scheme 
At the beginning of each $T_{C A}$, the queue length $q_{i}(t)$ of each VS will be measured and sent to the AP within the current $T_{C A}$, and based on that, the AP will allocate corresponding bandwidth to that queue in the following time interval. In [5], a HCCA-based closed-loop control model is proposed in order to drain the queue of each VS through controllable bandwidth allocation, and as a result the queuing delay could be minimized; the control model is given in Fig. 2.

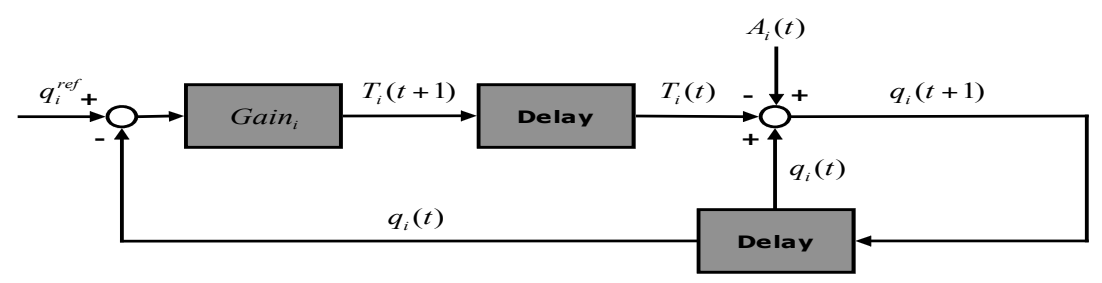

Fig. 2. The HCCA-based closed-loop control model of [5]

In Fig. 2, $q_{i}^{r e f}$ is a constant reference value set as a target queue length of VS $i ; q_{i}(t)$ is $i$ 's actual queue length at the beginning of the time interval $t$; Gain $_{i}$ is the control gain of the model to control the resource allocation in the following time interval $t+1 ; T_{i}(t)$ is the number of packets that can be transmitted related to the bandwidth allocation result and $A_{i}(t)$ is the number of packets that will arrive in the current interval. We see that $T_{i}(t+1)$ is directly determined by the gain and the differences between the target value $q_{i}^{r e f}$ and its current queue length $q_{i}(t)$, that is:

$$
T_{i}(t+1)=\operatorname{Gain} \times\left(q_{i}^{\text {ref }}-q_{i}(t)\right)
$$

The queue length at the beginning of interval $t+1$ is equal to that at the beginning of the previous interval $t$, plus the number of packets that will arrive and minus those that will be transmitted within $t$. This discrete-time linear model can be expressed as follow:

$$
q_{i}(t+1)=q_{i}(t)+A_{i}(t)-T_{i}(t)
$$

By applying this control algorithm, the required bandwidth at each VS can be dynamically allocated, hence, the number of packets remaining in the queue after each time interval is controlled and a maximum queuing delay can be guaranteed. However, there are still some limitations. First of all, the centralized behavior of the controller (AP) is not presented and only each individual virtual station is modeled and locally controlled. Also, the model can not be applied in saturated networks, although a bandwidth reallocation scheme in this condition is proposed. Their scheme may induce links transmitting at higher rates to be penalized more than those at lower rates; however, this is not always expected in real cases. For instance, a VS with higher traffic load and QoS requirements needs the priority to get more capacity but should not be penalized more than the others. In what follows, we will propose a new centralized control mechanism, so that more flexible and efficient bandwidth allocation can be achieved.

The simple control algorithm presented in this paper and the general control model used to analyze the system behavior is applicable to a range of wireless systems. Basically, we present an elementary resource allocation algorithm, and a control model for systems with a central scheduler, such as the UMTS HSPA (Universal Mobile Telecommunications System - High-Speed Packet Access) [6], IEEE 802.16d WiMAX (Worldwide Interoperability for Microwave Access) [7], and the IEEE 802.11e HCCA. The basic assumption is the presence of a central controller, and the ability for the wireless stations to signal their bandwidth requirements to the central controller. In this paper, we focus on the application to the IEEE 802.11e HCCA.

\section{A Centralized Control Model for Channel Resource Management}

In this section, we will present a centralized feedback control model for channel resource management based on the WLAN system defined in [5], and the relative advantages of it compared to that described above will be analyzed.

The discrete-time linear model described by (2) in essence describes the process block of our control system. Since in a real system the queue length $q_{i}(t+1)$ cannot assume negative values, an UnNeg function block should be added, which can be expressed as:

$$
\text { UnNeg Max }\left\{q_{i}(t+1), 0\right\} \text {. }
$$

Note that by the introduction of (3), the control model becomes non-linear. We adopt the same timing scheme as in [5], so that every VS within the network will measure the queue length at the beginning of each time interval $t$ and send the corresponding resource requirement within $t$, and then the AP will allocate the adapted channel resource to it in the 
following time interval $t+1$.

The resulting control model is illustrated in Fig. 3. Each VS $i(i=a, b)$ needs channel resources, managed by the AP, in each time interval to drain their queues, and each resource requirement, $R_{i}(t+1)$, is based on the comparison between the desired $q_{i}^{r e f}$ and the compensated actual queue length $q_{i}+A_{i}$, where $A_{i}$ is the number of arriving packets during one time interval:

$$
R_{i}(t+1)=K_{i} \times\left[q_{i}^{r e f}-q_{i}(t)-A_{i}(t)\right], \quad i=a, b,
$$

where $K_{i}$ is the gain of the control system; different values (for $K_{i}$ ) can be adopted based on different priorities of the VSs. For instance, a VS with higher QoS requirements should have higher priority to get more capacity to transmit; therefore, a higher gain (or lower $q_{i}^{\text {ref }}$ ) must be assigned to it. Normally, we desire to empty all the queues by assigning $q^{r e f}=0$ and vary control gains among VSs with different QoS requirements.

All the resource requirements from VSs in each time interval will be sent and then collected by the AP who plays the role of a Centralized System Coordinator (CSC). The AP will compare the Total Required Resources (TRR) with the Maximum of the Available channel Resource (MAR); the smaller one of these two is adopted as the total amount of resource to be allocated in that interval. On the other hand, the AP calculates the Resource Allocation Ratio (RAR) of each VS by multiplying the corresponding resource requirement $R_{i}$ with the inverse of the sum of the gathered requirements. Finally, fractions of the current available channel resource $R A_{i}$ will be derived based on the product of $R A R_{i}$ and the total amount of resource that can be allocated. The number of packets transmitted in time interval $t$ can then be expressed as:

$$
T_{i}(t)=R A_{i}(t) \times T R, \quad i=a, b,
$$

where TR is the physical layer data transmission rate.

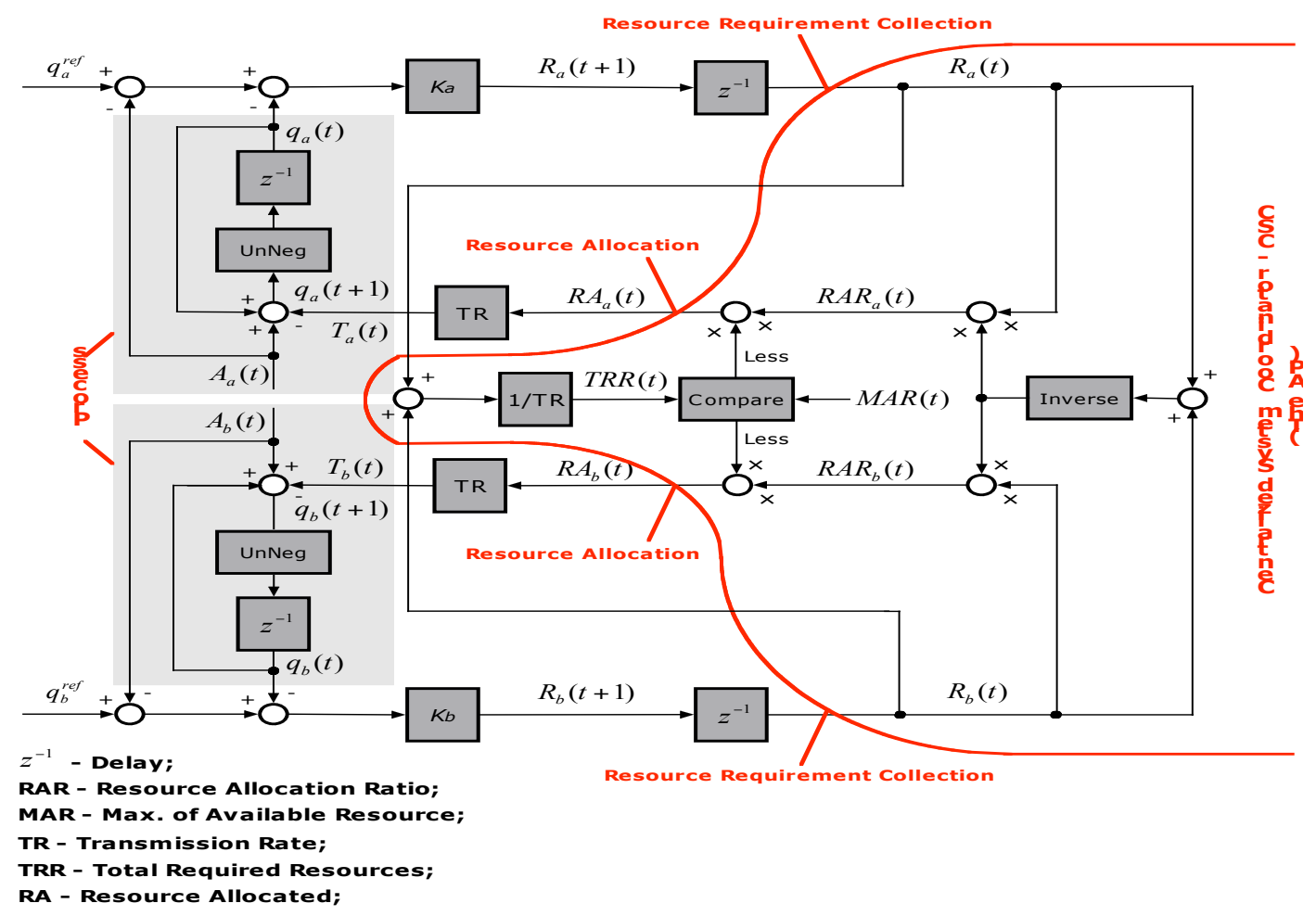

Fig. 3. The centralized access based control model for resource management

In Fig. 3, the right part represents the CSC. On the left side, each VS generates its new resource requirement $R_{i}$ based on the adapted $R A_{i}$ previously. Based on the description of our control model, the advantages compared to [5] are:

- The function of the central controller has been added to this model, which can provide management of the current available resources based on the gathered requirements.

- The dynamic resource allocation results for all the VSs within the system including their mutual interaction can be monitored by this control model.

- Flexible and efficient bandwidth reallocation can be achieved. According to the resource requirements collected by the controller AP, the current traffic load of the network can be judged. If the network is saturated, the AP will 
reallocate the limited channel resource and give relative more to the VSs with higher priorities in order to satisfy them. This can be achieved by assigning different control gains to these VSs.

\section{EXPERIMENTAL RESULTS}

In order to study the resource management model before applying it in practice, a software tool for modeling and analysis of control systems, called 20-sim [8], is used. The corresponding experimental results are presented below.

\section{A. Unsaturated Networks}

In this section, we assume the network is unsaturated, i.e., the TR is large enough to ensure that the TRR for every time interval is smaller than the MAR. There are two network scenarios considered.

1) Network Scenario 1: Two VSs $a$ and $b$ will send their data to the AP with constant bit rates $A_{a}$ and $A_{b}$ (the number of packets per time interval $t)$, respectively, and $T_{i}(i=a, b)$ denotes the number of packets allowed to transmit based on the resource allocation result in a certain interval, as shown in Fig. 4.
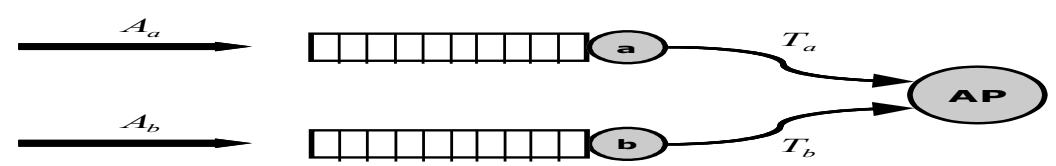

Fig. 4. Network scenario 1

The system parameters used to evaluate this scenario are listed in Table I. Note that the choice of most parameter settings is based on preliminary experiments and kept deliberately simple. We aim to drain the queues by setting the reference queue lengths to 0 . The duration of each time interval is set to be 1 s and within it, 20 and 10 packets will be generated by VS $a$ and $b$, respectively.

TABLE I

SYSTEM PARAMETER SPECIFICATION UNDER SCENARIO 1

\begin{tabular}{cc}
\hline Parameter & value \\
\hline$q_{a}^{r e f}$ & 0 \\
$q_{b}^{r e f}$ & 0 \\
$A_{a}(t)$ & $20 \mathrm{pkts} / \mathrm{s}$ \\
$A_{b}(t)$ & $10 \mathrm{pkts} / \mathrm{s}$ \\
$T_{C A}$ & $1 \mathrm{~s}$ \\
\hline
\end{tabular}

Based on the scenario and system parameter specification, two experiments with different system gains $K_{i}$ are designed to investigate the actual queue lengths $q_{i}$ at the beginning of each time interval $t$ and the number of packets being allowed to be transmitted within each interval based on the currently allocated channel resource $T_{i}$. The experimental results are shown in Fig. 5.
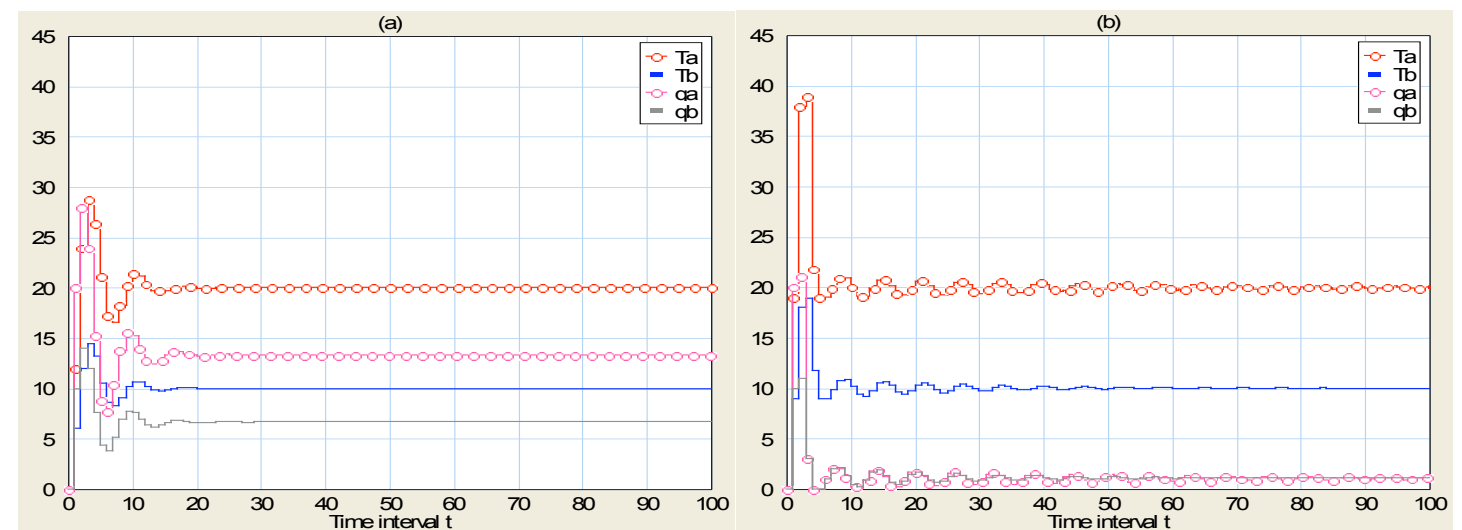

Fig. 5. (a) $T_{i}(t)$ and $q_{i}(t)$ obtained with gain settings $K_{a}=K_{b}=0.6$; (b) $T_{i}(t)$ and $q_{i}(t)$ with $K_{a}=0.95, K_{b}=0.9$

We see in Fig. 5(a) that after 20 time intervals, $T_{i}$ tends to settle, controlled by which the queue length $q_{i}$ reaches a stable level as well. From that point onwards, only a fixed amount of channel resource is required for the new incoming packets in each time interval. Since $K_{a}=K_{b}$, the proportion between $q_{a}$ and $q_{b}$ is equal to that between $A_{a}$ and $A_{b}$. It is easily seen that the target queue length is not reached for both $a$ and $b$, which means they still require more capacity. 
This can be achieved by further increasing the control gain $K_{i}$, as shown in Fig. 5(b). Note that the VS with larger $A_{i}(t)$ needs a higher gain to request more resources to drain its queue. Fig. 5 also shows that the overshoot and the settling time are enlarged when $A_{i}(t)$ and $K_{i}$ are increased. Hence, increasing the gains may be good for prioritization, whereas, it may have a negative effect as well: a certain amount of bandwidth may be wasted due to the over-reserving, especially in situations with highly fluctuating loads.

2) Network Scenario 2: In this scenario (illustrated in Fig. 6), a third VS $c$ is considered, which will forward the packets from both $a$ and $b$ (i.e., $A_{a}+A_{b}$ in total), and also transmit at the rate $A_{c}$ for itself. This scenario models the case where $c$ is a VS of the AP, which forwards packets received from regular stations towards other stations. The system parameters are specified as shown in Table II.

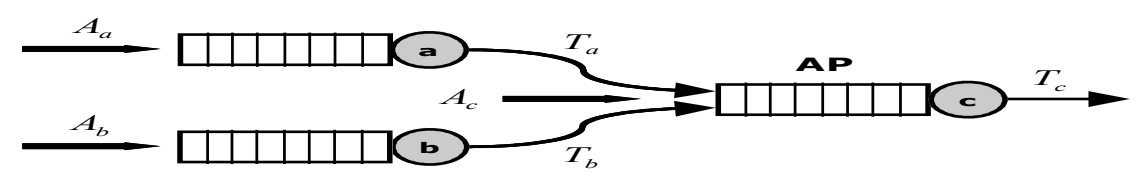

Fig. 6. Network scenario 2

TABLE II

SYSTEM PARAMETER SPECIFICATION UNDER SCENARIO 2

\begin{tabular}{ll}
\hline Parameter & Value \\
\hline$q_{a}^{r e f}$ & 0 \\
$q_{b}^{r e f}$ & 0 \\
$q_{c}^{r e f}$ & 0 \\
$A_{a}(t)$ & $10 \mathrm{pkts} / \mathrm{s}$ \\
$A_{b}(t)$ & $5 \mathrm{pkts} / \mathrm{s}$ \\
$A_{c}(t)$ & $10 \mathrm{pkts} / \mathrm{s}$ \\
$T_{C A}$ & $1 \mathrm{~s}$ \\
\hline
\end{tabular}

Since packets from $a$ and $b$ are injected into $c$, the overshoot of $q_{c}$ is larger than that of $a$ and $b$, and it will take longer for $q_{c}$ to stabilize, as shown in Fig. 7. Moreover, for VSs with larger $A_{i}(t)$ ( $i=a, b$ and $c$ ), a higher gain is required to approach the target queue length 0 . In Fig. 7(a), we see that all the VSs can approximately empty their queues. If $K_{a}$ and $K_{b}$ are further increased, as shown in Fig. 7(b), the influence on the overshoot (the overshoot of $q_{c}$ in Fig. 7(a) is around 28pkts and that in Fig. 7(b) is over 30pkts) and settling time of $q_{c}$ will be enlarged, whereas the stable state results of $q_{a}$ and $q_{b}$ are only improved slightly. Therefore, assigning proper gains to the VSs in the control system is considered to be important, which will be further investigated in our research.

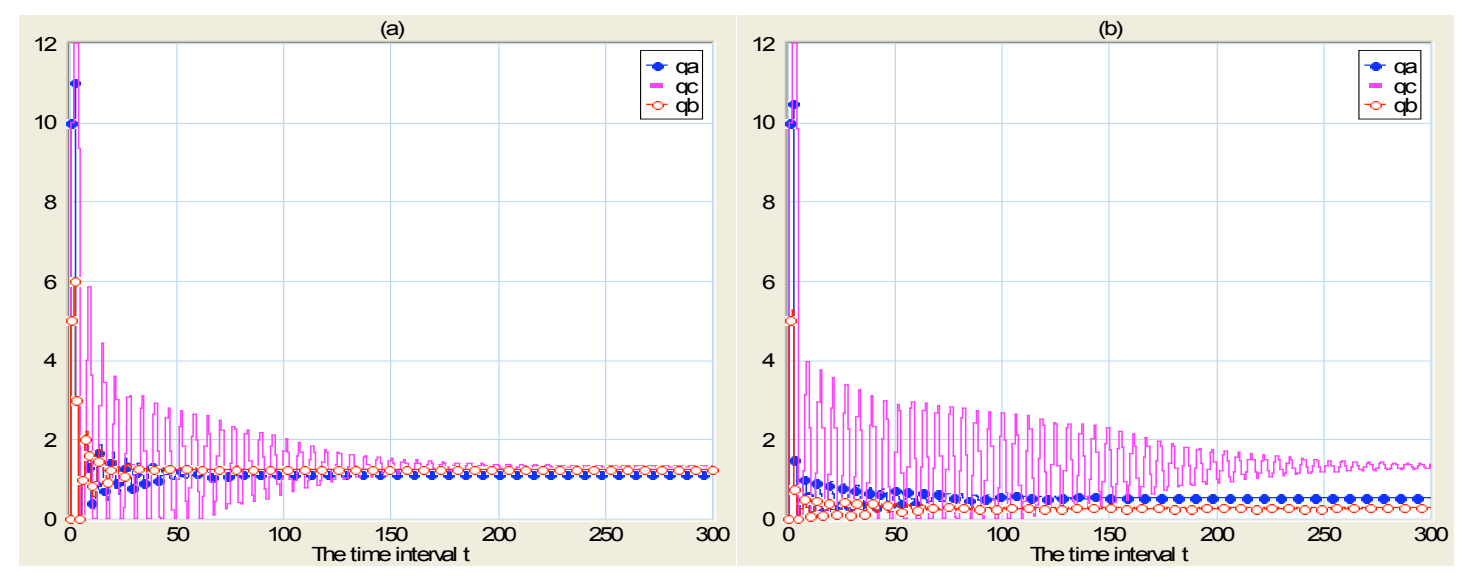

Fig. 7. (a) $T_{i}(t)$ and $q_{i}(t)$ obtained with gain settings $K_{a}=0.9, K_{b}=0.8, K_{c}=0.95$;

(b) $T_{i}(t)$ and $q_{i}(t)$ with $K_{a}=K_{b}=K_{c}=0.95$

\section{B. Saturated and Overloaded Networks}

Compared to the unsaturated network condition described above, experiments in this section are designed for Scenario 1 (Fig. 4) in saturated and overloaded networks.

As a first step, we adopt the system parameter specification as listed in Table I. According to the settings of $A_{a}(t)$, $A_{b}(t)$ and $T_{C A}$, we define the physical layer data transmission rate $T R$ to be $30 \mathrm{pkts} / \mathrm{s}$, thus, in each time interval, there are 30 packets arriving and the same amount can be served at most. We see in Fig. 8(a) that the fluctuation of $q_{i}$ and $T_{i}(i$ 
$=a, b)$ is smaller than that in unsaturated network condition, since the available resource here is limited, and the VSs can not obtain enough channel resources as requested in each time interval.

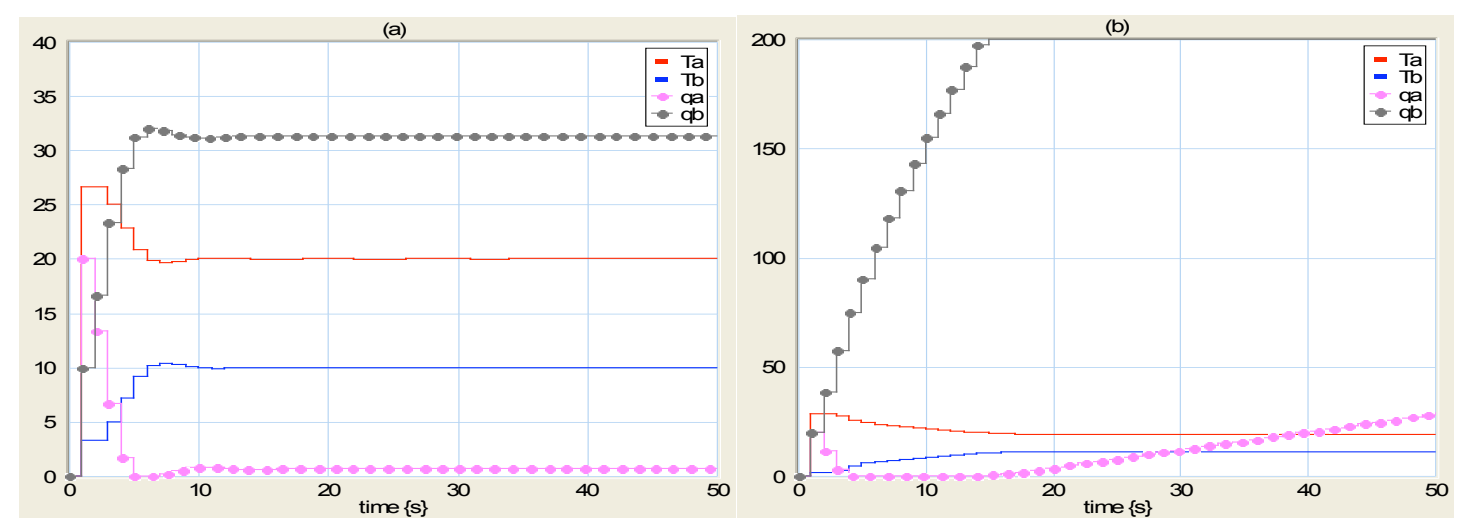

Fig. 8. (a) $T_{i}(t)$ and $q_{i}(t)$ obtained with gain settings $K_{a}=2.1, K_{b}=0.5$ in the saturated network;

(b) $T_{i}(t)$ and $q_{i}(t)$ with $K_{a}=10, K_{b}=0.5 \_$overloaded

Experiments show that by assigning a larger gain to the VS $a$ with higher QoS requirement, as shown in Fig. 8(a), the target queue length of $a$ is achieved although $A_{a}(t)>A_{b}(t)$. Furthermore, even in the overloaded network condition $\left(A_{b}\right.$ is reset to $20 \mathrm{pkts} / \mathrm{s}$ ), $a$ can still keep its desired queue length by further increasing $K_{a}$, whereas, $q_{b}$ will be built up as a tradeoff, which is presented in Fig. 8(b). We see that after 20 time intervals, $q_{a}$ starts to increase as well because more capacity is assigned to $b$ due to its increasing requirements. However, a VS should have a finite buffer size in practice, therefore $q_{b}$ will get restricted, as a result, the target queue length of $a$ can be maintained. Note that, compared to the unsaturated network condition, the channel resource is not sufficient for individual virtual stations in saturated and overloaded cases. Furthermore, since the resource allocation is related to the proportion of the assigned control gains, a larger gain can be adopted by the VS with a higher priority to increase its possession of the limited resource.

\section{Hybrid Networks with non-constant packet arrival rates}

We now study $T_{i}$ and $q_{i}$ of VSs $a$ and $b$ with non-constant packet arrival rates under the same system parameter specification as in section IV.B. As given in Fig. 9, the VS $a$ with a higher priority will send its data at the constant bit rate $20 \mathrm{pkts} / \mathrm{s}$. Within the first 50 time intervals, the network is unsaturated and afterwards, $A_{b}$ will be increased from 5 to $10 \mathrm{pkts} / \mathrm{s}$, and the network becomes saturated then.

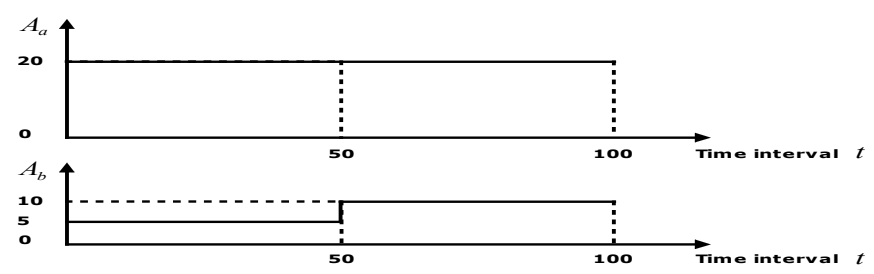

Fig. 9. Packet arrival rates of $a$ and $b$

Fig. 10 shows that, based on our specific control gain settings, the VS $a$ may drain its queue although $A_{a}$ is much higher than $A_{b}$ during the first 50 time intervals. After that, the background traffic $A_{b}$ is increased, which will break the current stabilization and the central controller AP will react on this case in the following control steps in order to arrive at a new stable state based on the current requirements from both $a$ and $b$. We see that $q_{b}$ is increased, related to the increase of $A_{b}$, which may cause an enlarged possession of the limited channel resource. As a result, $q_{a}$ slightly goes up. Note that the influence induced by the non-constant arrival rates can be further compensated by adjusting the gain of the control system if required. 


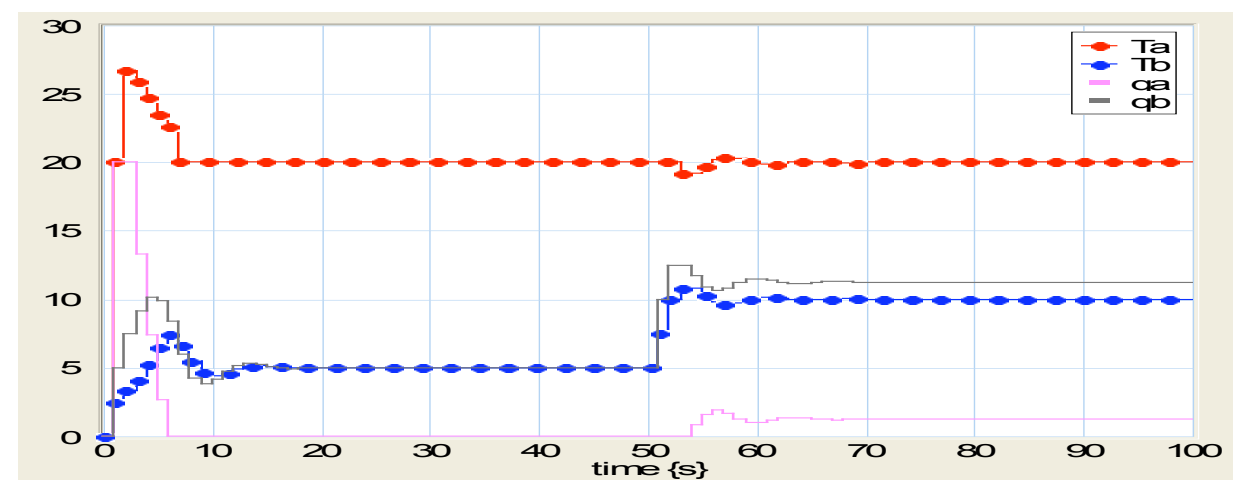

Fig. 10. $T_{i}(t)$ and $q_{i}(t)$ obtained with gain settings $K_{a}=1, K_{b}=0.5$

\section{CONCLUSION AND FUtURE WORK}

In this short paper, a centralized feedback control model for resource management has been proposed and adapted to a specific application for WLANs with a centralized medium access method. We have studied the model by using the software package 20-sim and the experimental results have shown that it is able to provide flexible and efficient resource allocation based on current gathered requirement information under our specified network scenarios and different traffic load conditions. More scenarios have already been studied.

Our future research will focus on adaptable gain allocation algorithms in dynamic network environments, studying the non-linearity of control systems, testing and adapting the control model by considering the wireless nature of the medium (e.g., random losses, fluctuating traffic, etc.) and embedding the improved model in detailed discrete-event simulation. Among others, we plan to extend the model to the fully distributed case (i.e., the IEEE 802.11 EDCF), to model multi-hop ad-hoc networks. Extension of the model, to incorporate random packet arrivals is also foreseen.

\section{REFERENCES}

[1] A. Sampath, P. S. Kumar and J. Holtzman, "Power control and resource management for a multimedia CDMA wireless system", in Proc. IEEE PIMRC'95, Vol. 1, pp. 21-25.

[2] I. Cardei, S. Varadarajan, A. Pavan, L. Graba, M. Cardei and M. Min, "Resource management for ad-hoc wireless networks with cluster organization", Journal of Cluster Computing in the Internet, Vol. 7, No. 1, pp. 91-103, Jan. 2004.

[3] Richard C. Dorf and Robert H. Bishop, "Modern control systems", Eighth Edition, 0-201-32677-9.

[4] W. H. Kwon and H. S. Kim, "A survey of control theoretic approaches in wired and wireless communication networks", Korea-Japan Joint Workshop 2000, Vol. 1, No. 1, pp. 30-45, Aug. 2000.

[5] G. Boggia, P. Camarda, L. A. Grieco and S. Mascolo, "Feedback-based bandwidth allocation with call admission control for providing delay guarantees in IEEE 802.11e networks", Computer Communications, Vol. 28, No. 3, pp. 325-337, Feb. 2005.

[6] A. Masmoudi, D. Zeghlache and S. Tabbane, "Resource and scheduling optimization in HSDPA based UMTS networks", $6^{\text {th }}$ World Wireless Congress 2005 (WWC'05), San Francisco, May 2005.

[7] Y. Xu, Z. Li, X. Zhong and H. Xu, "A bandwidth scheduling algorithm based on minimum interference traffic in mesh mode", in Proc. IEEE WiCOM’07, pp. 822-825, Sep. 2007.

[8] http://www.20sim.com/. 